\title{
Modular System Approach for Modelling Socio-Technical Transitions towards Alternative Energy Infrastructures in Urban Areas
}

\author{
$\underline{\text { A. Rojas }}^{\text {a }}$, F. de Haan ${ }^{\mathrm{b}}$, S. Candy ${ }^{\mathrm{b}}$ and L. Aye ${ }^{\mathrm{a}}$ \\ ${ }^{a}$ Renewable Energy and Energy Efficiency Group, Department of Infrastructure Engineering, Melbourne \\ School of Engineering. The University of Melbourne, Victoria, Australia, 3010. \\ ${ }^{\mathrm{b}}$ Melbourne School of Design, The University of Melbourne, Victoria, Australia 3010. \\ Email: angelara@student.unimelb.edu.au
}

\begin{abstract}
Reducing greenhouse gas emissions (GHGE) in urban energy systems requires the implementation of alter-native infrastructure configurations across different geographical, technical and social scales. Furthermore, alternative configurations may improve systems resilience and democratization of service provision. How-ever, unlike current centralised systems, which are well understood from the technical and social perspectives, there is a lack of knowledge on the socio-technical interplay across multiple intermediate scales of alternative infrastructures, defined as all the possible configurations in between offgrid and centralised infrastructure. There are various concepts in the literature describing decentralised, distributed and integrated systems using different primary energy resources and generating various energy carriers -for electricity, heating, and cool-ing. However, the information on alternative models is limited to the technical requirements leaving a gap in knowledge on the societal requirements. Alternative infrastructure models potentially reduce GHGE but they may require unique forms of social organisation structures to support their adoption and increase the pace towards decarbonisation. To obtain in-depth understanding of the socio-technical interplay of alternative in-frastructures across scales, this paper draws on complexity theory, the concept of modularity and transitions modelling literature. This aid in the definition of the proposed conceptual framework and the Service Provision Modules (SPM). This conceptual framework serves as the basis for spatially specific modelling and simula-tion. The SPM may use different types of networks and can represent any type of conventional or alternative infrastructure configuration. The conceptual framework then uses the modules to construct the socio-technical layouts for the baseline and possible future configurations in a given area. The paper briefly outlines various concepts in the literature which technically define alternative energy systems, then presents a description of the conceptual framework and the definition of an SPM. Finally, the SPMs are used to represent the Australian electricity system.
\end{abstract}

Keywords: $\quad$ Socio-technical systems, synergies, transitions, modelling, urban 
Rojas et al., Modular System Approach for Modelling Socio-Technical Transitions towards alternative...

\section{INTRODUCTION}

There is an urgent need to decarbonise cities. Up to $44 \%$ of global energy related $\mathrm{CO}^{2}$, a significant greenhouse gas (GHG), is contributed by urban areas. And $70 \%$ of this GHG contribution, comes only from final energy use [Wu et al., 2015]. Also, cities are particularly vulnerable to climate change impacts, therefore cities must intensify their autonomy, flexibility, diversity and adaptability, which in turn improve its resilience. Furthermore, urban services of provision could benefit from diverse configurations, such a distributed system models. This enable their democratization [Biggs et al., 2009] in a way that ensures environmental and social justice [Wolsink et al., 2013]. It is clear that existing centralised systems will not be adequate enough to address these challenges [Bouffard and Kirschen, 2008], therefore, alternative forms of service provision must be explored.

Traditional centralised infrastructure is well understood, both technically and organisationally, however, there is a lack of understanding on the socio-technical requirements of alternative models which often operate on different scales and use a variety of technological and societal configurations. Existing research proposes many alternative models of energy provision, that typically have one or a combination of properties for distributed, integrated, and decentralised system models. However, the majority of studies focus on proposing specific technical or infrastructural solutions [Alanne and Saari, 2006; Mancarella, 2014; Manfren et al., 2011; Mohan et al., 2016; Larsson et al., 2011].Although this is essential to prove their viability, technical solutions must operate within a societal or organisational context and their requirements to support technical solutions are lacking.

Since energy generation and use accounts for a high proportion of GHGE, intervention in urban energy systems is a key area to address decarbonisation, democratization and resilience of service provision in cities. Therefore, this paper investigates energy systems, electricity to be precise, as a starting point and develops a conceptual framework to map, model and simulate various aspects of electricity provision, to enable investigation of impacts on GHGE from alternative infrastructures across multiple geographical, technical, and social scales. These alternative infrastructure are represented as socio-technical layouts each is one instance of a set of organisations and technologies in a chosen area given its demographics, resource availability and demand profiles.

This study also defines the service provision module (SPM) used by the conceptual framework. These modules are capable of representing, in principle, any system for energy provision -traditional, decentralised, and other alternative models. Furthermore, each technical representation of a system of energy provision will be accompanied by social structures (e.g. modes of organisation) across various scales. To achieve this, the framework employs a modular approach for the representation.

The framework draws on previous research from various disciplines, notably engineering, complex systems, networks, modular systems theory and sustainability transitions studies, particularly transitions modelling. In Section 2, brief description on alternative infrastructures for energy systems is presented. Section 3 describes the service provision modules and the conceptual framework. Section 4 presents an example of how the sociotechnical modules can represent different urban energy infrastructures using the Australian electricity system as a case study. Finally, conclusions and recommendations for future work are presented in Section 5.

\section{BaCkground: Alternative Energy Models}

Technological advancements on energy systems are progressively giving rise to diverse technical models and concepts such as Distributed Generation (DG) [Alanne and Saari, 2006; Pepermans et al., 2005], Decentralised Energy Systems (DES), Distributed Energy Resources (DER), Distributed Multi-generation (DMG) [Chicco and Mancarella, 2009], Multi-energy Systems (MES) [Mancarella, 2014], Integrated Energy Systems (IES) [Manfren et al., 2011; Lin and Bie, 2016], Integrated Community Energy Systems (ICES) [Mendes et al., 2011], Hybrid DMG, Polygeneration [Liu et al., 2007], and Synergy Grids [Larsson et al., 2011].

The specific definition and description on characteristics of these models are outside the scope of this paper. These models, however, have in common the possibility to be configured across specific scales and can integrate different services of provision. Additionally, they range from stand alone self-managed configurations to distributed system models; may include demand response applications and/or storage; may generate more than one energy carrier -for electricity, heating, and cooling and; can use a variety of energy resources for the generation and conversion process, including renewable energies, fossil fuels, or waste.

The concepts of Smart Infrastructures, Smart Grids and Smart-microgrids build upon existing alternative models but incorporate Information and Communication Technologies (ICTs) to manage the grid more efficiently. These ICTs can be used in demand response applications, and disaster management. Besides specific com- 
munication protocols, these concepts include sensors, smart meters, bidirectional power converters, and smart appliances.

Urban infrastructures, considered societal systems functioning to meet societal needs [de Haan and Rotmans, 2011] are currently failing on this objective and notably, none of the technical concepts described specify the corresponding social structure requirements. Scholars have covered intervention methodologies for the introduction of smart microgrid systems in rural communities based on a socio-ecological system approach [Alvial-Palavicino et al., 2011], while others have assessed how trust plays a vital role on the dynamics and outcomes of community renewable energy projects [Walker et al., 2010], or analysed how to achieve an equitable distribution of power-generating capacity in microgrids [Wolsink et al., 2013]. This literature is valuable when analysing social construction for alternative energy system, however, it is often focused on one specific scale and grid configuration narrowing down the possibilities for other socio-technical solutions.

\section{Modules and Conceptual Framework}

To determine the framework, this study draws on both, complex systems and modular systems theories. Additionally, the conceptual framework builds upon the transitions modelling field as the framework guides a model and simulation to analyse the potential transition pathways towards technical configurations and their societal requirements that will enable the decarbonisation and democratization of systems for energy provision.

First, a complex system can be considered a large network of relatively simple individual components or functional roles, in which emergent complex behaviour is exhibited [Mitchell, 2006]. Complex systems have agents dynamically interacting through networks exhibiting self-organisation, emergence, path-dependency and co-evolution properties. Additionally, complex (adaptive) systems can learn and adapt through different processes. According to these properties, there is a synergistic relationship between complex systems theories and energy systems [Bale et al., 2015].

Second, modular systems theory facilitates the analysis of a system by governing the extent to which a system's components can be separated and recombined, and the degree to which the rules of the system's architecture enable or constraint the combination and matching of components [Schilling, 2000]. The study of the individual components of the complex system for energy provision helped to determine a generic energy provision module. the SPM, that could represent any configuration including alternative infrastructure models.

Finally, modelling and simulation of socio-technical systems have been widely used by the transitions modelling community (see [Bergman et al., 2008; Chappin, 2011; Haxeltine et al., 2008]), which has advance the understanding on complexity and dynamics of socio-technical change. Therefore, this work builds upon transitions modelling contributing to the field with a generic modular approach for socio-technical representations of systems for service provision.

\subsection{Socio-technical Modules for Service Provision}

Systems for energy provision, specifically in the electricity case, usually consist of elements for power generation and conversion, transmission and distribution networks, energy retailers and providers, and end users. Each of these systems can incorporate a variety of technologies, business models and societal structures. Additionally, depending on the generation technology used and the energy demand, systems for energy provision affect the natural environment with GHGE and intensive resource depletion.

Large scale power plants can use a variety of primary energy sources (e.g. fossil fuels, wind, and solar) depending on the generation technologies, which may include turbines and generators coupled with transformers. Electricity generated is then aggregated on the main grids using poles and wires to be distributed to end users.

Conversely, decentralised or stand-alone systems use small generation units (SGU) to generate electricity. These SGU may be rooftop solar PV panels, small wind turbines, micro-hydro systems, and diesel generators. The distribution network in a stand-alone system consist of the internal electrical connections of individual building. Additionally, any system configuration may use different types energy storage technologies, mechanical, gravitational, and electrochemical, to reduce transmission losses and improve reliability of supply.

Arguably, distributed systems (DS) range in between centralised and decentralised generation. Independently from the power capacity rating, the area of power delivery, the technology used, the environmental impact, and the ownership, Distributed Generation (DG) in electricity provision is defined as an electric power source where the units of generation are located - installed and operated- close to the loads. This means that the generation is connected directly to the distribution network or on the customer site of the meter [Ackermann et al., 2001]. 
From the previous background and the brief descriptions of the different energy system configurations presented, this paper defines the service provision module (SPM) as the individual components of an energy system. SPMs are used in this study to represent any system configuration for electricity generation, but they may be used to represent any type of service provision such as water, food, and waste and the synergies of different systems to generate a service, for instance, from waste to energy.

An SPM (see Figure 1) has the generation and conversion for service provision (GSP), a grid, optional storage and the interface (I). Additionally, SPMs have a societal structure attached to it, which represents the societal requirements or characteristics of certain configurations. The GSP do not necessarily exist physically on the study area, taking a consumption based approach, if the area under study is supplied by a distant GSP, its generation is considered virtual in the module. The grid in the SPM is used to represent different types of networks and their characteristics (e.g. transmission and distribution). The storage is optional, it can vary depending on physical characteristics, capacity, life-cycle, charging/discharging time, and costs. The interface is the point where the SPM interacts with the demand. Depending on the desired scale of analysis, the interface can be a large industrial area, a substation in a neighbourhood, or the internal power supply grid of a building. Notably, the SPMs are recursive as the surplus of energy or waste can be (re) used in other module as input for a GS

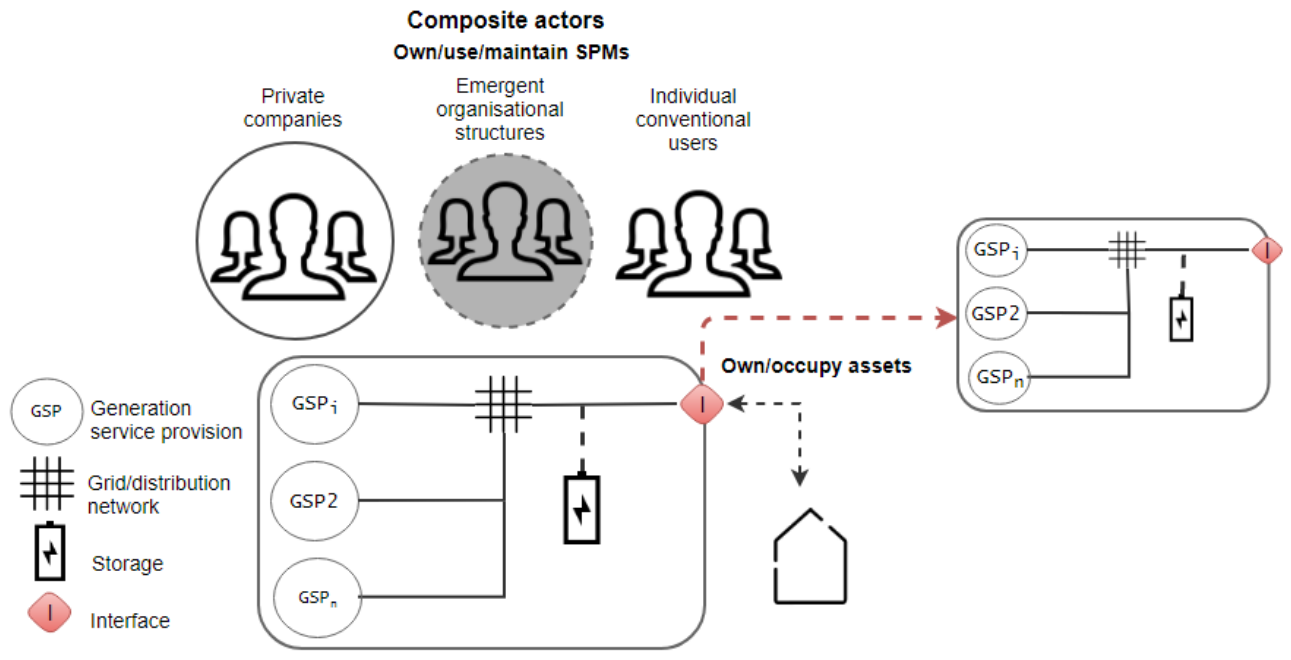

Figure 1. Service Provision Module (SPM): Technological configuration and composite actors.

Additionally, Figure 1 shows the societal structures as composite actors. These agents can own, use and/or maintain an SPM. Individuals can have different roles and can subscribe to more than one role, for instance, a person can be a household and member of a cooperative at the same time. Other roles include organisational ones, such as private companies. Emerging social structures (e.g. cooperatives) are represented in the middle with the actors in the grey area. These emerging structures may represent prosumer models such as peerto-peer, prosumers connected to microgrids, prosumer-to-islanded microgrids and organised prosumer groups [Parag and Sovacool, 2016]. The composite actors in the right-hand side of Figure 1 represent individual conventional users. On the demand side, composite actors can be analysed depending on ownership and/or occupancy of certain assets -houses, offices, and public b uildings. This distinction is made to calculate the demand profile, which not only depends on the end u ser's consumption but also on the type of building the user occupies in the case study area.

Therefore, the societal representation associated to the SPM depends on the institutional delineation of the system on the case study area. Moreover, this definition of composite actors allows the representation of not only a conventional individual end users but also groups of end users, prosumers or producers, depending on their roles at the scale of the system under study.

\subsection{Description of Conceptual Framework}

As shown in Figure 2, the conceptual framework has various input, with external and internal data, and processing layers. Within the initialisation layers, a first layer for spatial representation of the area under study with data related to weather conditions such as global horizontal irradiance (GHI), temperature, wind speed, and relative humidity. A second layer has demographic and land use data for the selected area. Then, there are two libraries with the information about different technologies for the SPM and different modes of organ- 
isation, characteristics and requirements for the composite actors. The initialisation layers are then used to

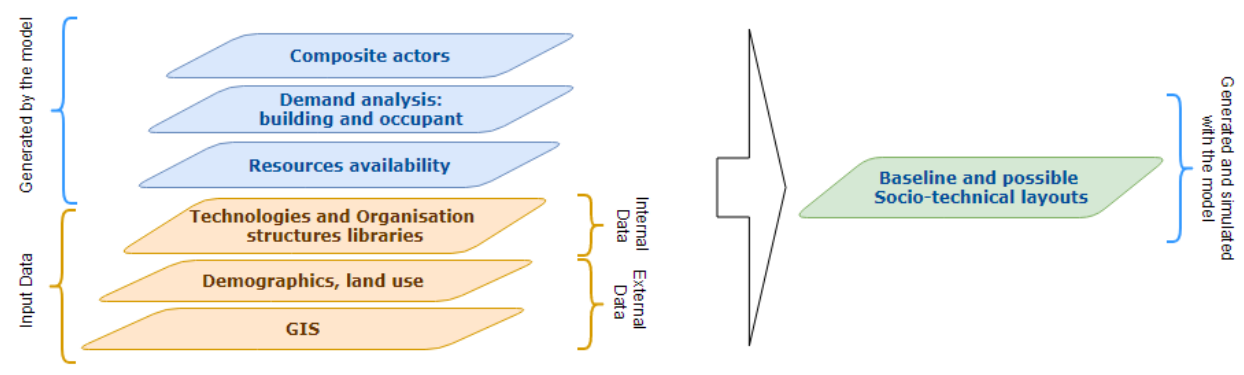

Figure 2. Conceptual Framework (CF).

calculate for the area under study the resources availability, demand analysis and the type of composite actors for the area selected for the study. The "Resource Availability" layer generates the fuel availability and reliability for electricity generation. For instance, data on GHI as primary energy source availability, and fossil fuel reserves. SPMs can find barriers or enablers for the uptake of certain alternative configurations depending the "resource availability" information. This layer also shows the surplus of any other type of energy flow that can be reused as "primary" energy resource by another GSP.

Composite actors have a demand. Their demand depends on their activities and the space they occupy. Therefore, the "Demand Analysis" layer has two main components: physical space and consumer based demand. To clarify, the purpose of the framework is not to optimize supply for a given demand with technological solutions but to explore alternative configurations of service provision. Therefore, the assumptions and data used in the analysis alternative configurations are based on technical systems proven to be successful when underpinned by certain social infrastructures. Finally, the "Composite actors" layer uses the societal unites attached to the SPM and the input data on demographics and organisation structures to identify the composite actors within the area under study.

\section{SOCIO-TECHNICAL REPRESENTATION OF URBAN ENERGY SYSTEMS WITH SERVICE PROVISION MODULES}

This section uses the SPM to represent the different configurations of the current system for electricity provision described by the Australian Energy Regulator. Additionally, it shows where novel social structures may emerge and how the surplus of energy or waste can be used by other SPM as resource for energy generation.

Figure 3 shows a modified version of the electricity supply chain described by The Australian Energy Regulator (AER). This supply chain starts with the production of energy by the generators, from a variety of sources using diverse technologies. Part (a) of Figure 3 uses generation from large scale power plants $\left(G S P_{L S i}, \ldots, G S P_{L S n}\right)$, steps up the electricity output to be transported over large distances using the transmission network. Large industries may connect to the interface point and use electricity directly from transmission networks. Part (b) shows the SPM using the distribution network, the interface point checks first in with the intermediaries between energy generators and energy users, who can be the energy authorised retailers or other energy onsellers.

The composite actors associated to the SPM of Figure 4 (a) and (b) are, most likely, private companies who own and maintain large scale power plants and parts of the transmission and/or distribution networks. Part (c) represents current alternative electricity providers, who install solar PV panels or other small-scale generators at a customer's premises and sell output to the customer or other customers. In (c) generation is represented with "generators of alternative service provision" using different small-generation units (SGU) or $\left(G A S P_{S G U i}, \ldots, G A S P_{S G U n}\right)$. The electricity supply representation from the AER is modified as it includes in (d) standalone system with SGU, electric vehicles as storage and an individual grid; or a prosumer connected to a microgrid.

As an example in Victoria, Australia, to be eligible, SGU capacity must be of no more than $100 \mathrm{~kW}$ for a solar PV array, $10 \mathrm{~kW}$ for a wind power system and $6.4 \mathrm{~kW}$ for a hydro power system. According to the Clean Energy Regulator, the certificates for SGU are given to individuals and small businesses. Therefore, this could be represented with an off-grid SPM with a composite actor who owns, uses and maintains it. This composite actor can be an individual or a small business who either owns, occupies -or both, an asset -the building- where the electricity generated by the SGU is used. 
Rojas et al., Modular System Approach for Modelling Socio-Technical Transitions towards alternative...

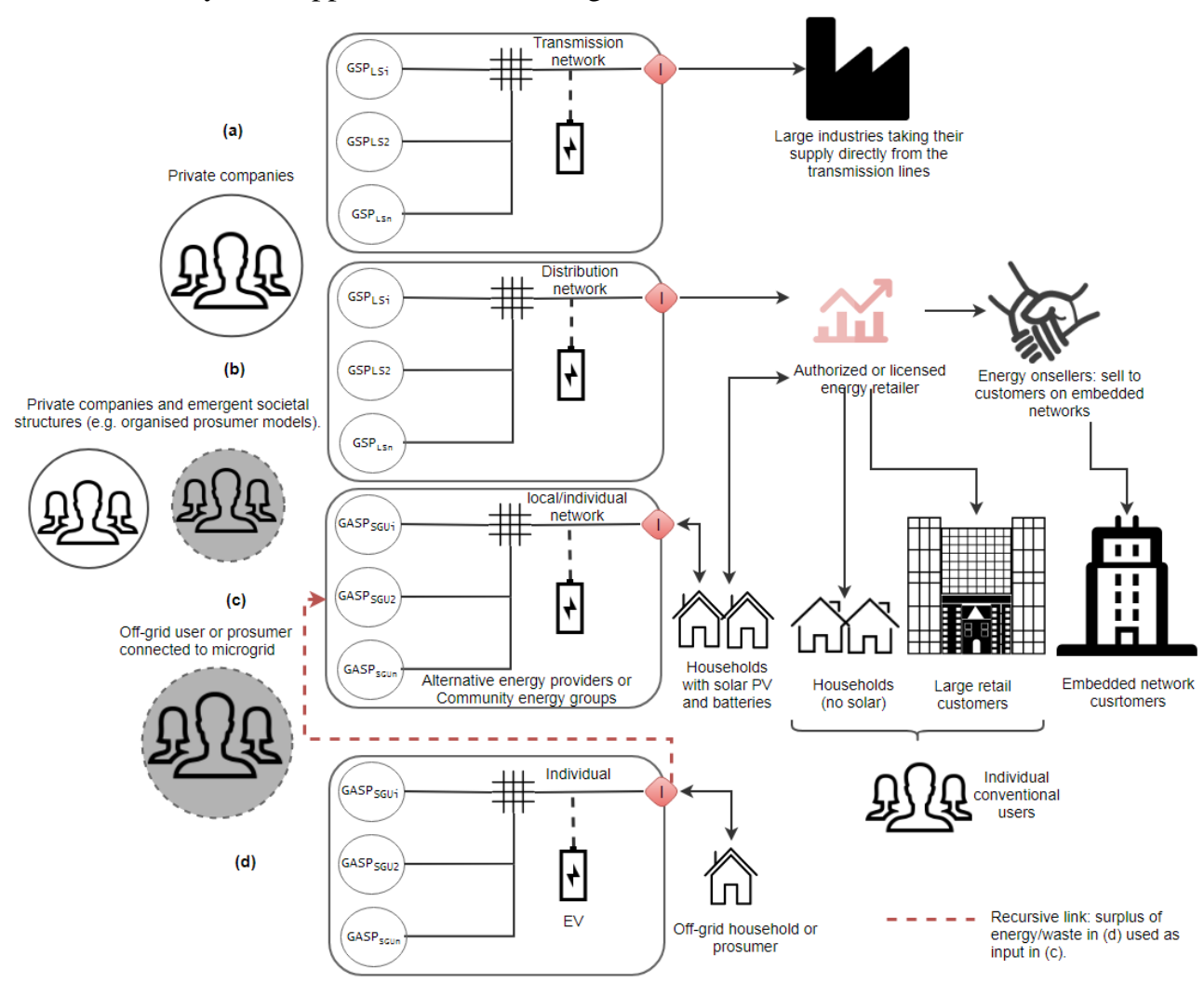

Figure 3. (a) Centralised large scale power plants owned by private companies connected to large industries through the transmission network; (b) energy retailers or other intermediaries; (c) alternative energy providers;(d) stand alone system or prosumer to microgrid.

\section{CONCLUSIONS AND FUTURE WORK}

There is a need for alternative infrastructures for service provision to decarbonise, democratise and improve the resilience of systems for service provision in urban areas. Traditional, centralised infrastructures are well understood from technical and societal perspectives, however, alternative infrastructures lack a better understanding on the societal requirements for their adoption. Moreover, the interplay between alternative sociotechnical systems across scales is also poorly understood. Therefore, this paper proposes a conceptual framework to find the baseline and possible alternative systems for service provision, specifically electricity, for a given area. The conceptual framework developed uses the service provision modules to represent any energy system with a modular approach. The framework developed has the GIS, demographics and land use data as external input data and technologies and organisation structure libraries as internal input data in the initialisation layers. Based on the framework developed, a model should generate the resource availability, demand and type of composite actors for the area under study. The model also calculates the baseline socio-technical layout and explore possible future socio-technical layouts. Finally, the paper presents how the service provision modules can represent different energy system configurations within the current electricity supply chain of Australia.

Future work should be focus on identifying and describing the specific modelling methods and techniques to obtain the socio-technical layouts following the conceptual framework developed for both, the baseline and future configurations for service provision. It is anticipated that the conceptual model presented could benefit from methods such as spatial microsimulation and agent based modelling. There has been significant development on spatial microsimulation in Australia [Tanton et al., 2011; Brown and Harding, 2002]. However, the spatial microsimulation research is been mainly focused on areas of health -diabetes, obesity-, public economic policy -taxation, social security, welfare- and superannuation schemes, leaving other areas related to services of provision, such as energy, largely unexplored with these methods. Therefore, the conceptual model presented can greatly take advantage from spatial microsimulation methods, for instance, in the demand analysis layer. Finally, efforts should be made to find appropriate methods for the assessment of the transformative potential for a case study area with the conceptual framework and the applicability and effectiveness of various policy interventions. 
Rojas et al., Modular System Approach for Modelling Socio-Technical Transitions towards alternative...

\section{REFERENCES}

Ackermann, T., G. Andersson, and L. Söder (2001). Distributed generation: a definition. Electric power systems research 57(3), 195-204.

Alanne, K. and A. Saari (2006). Distributed energy generation and sustainable development. Renewable and sustainable energy reviews 10(6), 539-558.

Alvial-Palavicino, C., N. Garrido-Echeverría, G. Jiménez-Estévez, L. Reyes, and R. Palma-Behnke (2011). A methodology for community engagement in the introduction of renewable based smart microgrid. Energy for Sustainable Development 15(3), 314-323.

Bale, C., L. Varga, and T. Foxon (2015). Energy and complexity: New ways forward. Applied Energy 138.

Bergman, N., L. Whitmarsh, and J. Köhler (2008). Transition to sustainable development in the UK housing sector: from case study to model implementation. pp. 32.

Biggs, C., C. Ryan, J. Wiseman, and K. Larsen (2009). Distributed Water Systems :. (2), 1-31.

Bouffard, F. and D. S. Kirschen (2008). Centralised and distributed electricity systems. Energy Policy 36(12).

Brown, L. and A. Harding (2002). Social modelling and public policy: Application of microsimulation modelling in australia. Journal of Artificial Societies and Social Simulation 5(4).

Chappin, É. J. L. (2011). Simulating energy transitions. Next Generation Infrastructures Foundation.

Chicco, G. and P. Mancarella (2009). Distributed multi-generation: a comprehensive view. Renewable and sustainable energy reviews 13(3), 535-551.

de Haan, F. and J. Rotmans (2011). Patterns in transitions: understanding complex chains of change. Technological Forecasting and Social Change 78(1), 90-102.

Haxeltine, A., L. Whitmarsh, N. Bergman, J. Rotmans, M. Schilperoord, and J. Köhler (2008). A Conceptual Framework for transition modelling. Int. J. Innovation and Sustainable Development 3(1/2), 93-114.

Larsson, N., S. Salat, L. Bourdic, and F. Hovorka (2011). From smart grids to synergy grids. In Proceedings of the World Sustainable Building Conference.

Lin, Y. and Z. Bie (2016). Study on the Resilience of the Integrated Energy System. Energy Procedia.

Liu, P., D. I. Gerogiorgis, and E. N. Pistikopoulos (2007). Modeling and optimization of polygeneration energy systems. Catalysis Today 127(1-4), 347-359.

Mancarella, P. (2014). Multi-energy systems: An overview of concepts and evaluation models. Energy 65.

Manfren, M., P. Caputo, and G. Costa (2011). Paradigm shift in urban energy systems through distributed generation: Methods and models. Applied Energy 88(4), 1032-1048.

Mendes, G., C. Ioakimidis, and P. Ferr??o (2011). On the planning and analysis of Integrated Community Energy Systems: A review and survey of available tools. Renewable and Sustainable Energy Reviews 15(9).

Mitchell, M. (2006). Complex systems: Network thinking. Artificial Intelligence 170(18), 1194-1212.

Mohan, G., M. Kumar, and A. Martin (2016). Solar thermal polygeneration system for cooling, fresh water, and domestic hot water supply: Experimental analysis. In Renewable Energy in the Service of Mankind Vol II, pp. 781-791. Springer.

Parag, Y. and B. K. Sovacool (2016). Electricity market design for the prosumer era. Nature Energy 1, 16032.

Pepermans, G., J. Driesen, D. Haeseldonckx, R. Belmans, and W. D'haeseleer (2005). Distributed generation: definition, benefits and issues. Energy policy 33(6), 787-798.

Schilling, M. A. (2000). Toward a general modular systems theory and its application to interfirm product modularity. Academy of management review 25(2), 312-334.

Tanton, R., Y. Vidyattama, B. Nepal, and J. McNamara (2011). Small area estimation using a reweighting algorithm. Journal of the Royal Statistical Society: Series A (Statistics in Society) 174(4), 931-951.

Walker, G., P. Devine-Wright, S. Hunter, H. High, and B. Evans (2010). Trust and community: Exploring the meanings, contexts and dynamics of community renewable energy. Energy Policy 38(6), 2655-2663.

Wolsink, M. et al. (2013). Fair distribution of power generating capacity: justice, microgrids and utilizing the common pool of renewable energy. Energy Justice in a Changing Climate. Social equity and low-carbon energy.

Wu, L., G. Broquet, P. Ciais, V. Bellassen, F. Vogel, F. Chevallier, I. Xueref-Remy, and Y. Wang (2015). Atmospheric inversion for cost effective quantification of city co 2 emissions. Atmospheric Chemistry \& Physics Discussions 15(21). 